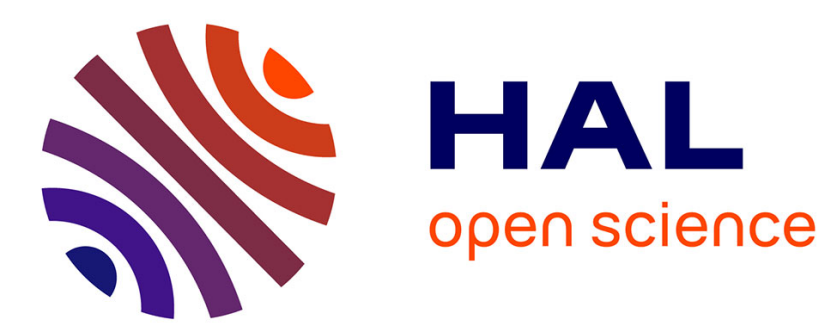

\title{
Les phrases [si P, q] ou la stratégie de l'alternative dans deux proverbes de Musset
}

Véronique Magri-Mourgues

\section{To cite this version:}

Véronique Magri-Mourgues. Les phrases [si P, q] ou la stratégie de l'alternative dans deux proverbes de Musset. L'information grammaticale, 2012, 135, pp.3-9. hal-01226780

\section{HAL Id: hal-01226780 \\ https://hal.science/hal-01226780}

Submitted on 10 Nov 2015

HAL is a multi-disciplinary open access archive for the deposit and dissemination of scientific research documents, whether they are published or not. The documents may come from teaching and research institutions in France or abroad, or from public or private research centers.
L'archive ouverte pluridisciplinaire HAL, est destinée au dépôt et à la diffusion de documents scientifiques de niveau recherche, publiés ou non, émanant des établissements d'enseignement et de recherche français ou étrangers, des laboratoires publics ou privés. 


\section{LES PHRASES [SI P, Q] OU LA STRATEGIE DE L'ALTERNATIVE DANS DEUX PROVERBES ${ }^{1}$ DE MUSSET}

Le corpus des deux Proverbes de Musset fournit un ensemble de 135 occurrences de si, autres que l'adverbe intensif. L'extraction des occurrences a été réalisée automatiquement et portent sur la forme $s i$ ou la forme élidée $s$ '; le retrait des exemples de $s i$ adverbe intensif a été fait manuellement. En dépit de l'analyse de G. Moignet ${ }^{2}$ qui relie les divers emplois de si par une dynamique subductive, depuis l'état plénier de l'adverbe d'affirmation jusqu'à l'état le plus subduit de la conjonction hypothétique, en passant par les cas de l'adverbe, de degré ou exclamatif, ou encore le cas du marqueur de l'interrogation indirecte, je considère que le fonctionnement de $s i$ adverbe intensif relève d'une analyse autre que celle qui est entreprise ici.

L'enjeu de cet article est d'articuler analyse linguistique et interprétation stylistique, en étudiant comment les propriétés des propositions en si sont exploitées dans les deux Proverbes de Musset d'une part et comment, d'autre part, elles peuvent être interprétées pour en découvrir les enjeux argumentatifs ou dramatiques et y trouver des révélateurs du regard narquois de Musset sur les relations humaines et sur une certaine manière d'envisager la vie.

Ces exemples attestés et, de fait, contextualisés, ont été d'abord catégorisés selon l'effet de sens produit par la combinaison [si p, q], qui repose sur un schème corrélatif ${ }^{3}$. Une première approche de ces constructions établit un partage entre systèmes hypothétiques ou conditionnels et systèmes dits non conditionnels ou factuels. En parcourant la liste des occurrences fournies par le corpus d'étude, on voit qu'une lecture unifiée de ces deux systèmes, proposée par certains théoriciens, est cependant possible. L'approche sémantique de [si p, q] propose d'y lire l'instauration d'un mouvement suspensif, proche de l'interrogation, et la construction de mondes alternatifs. Enfin, l'interprétation de ces systèmes corrélatifs peut être évaluée, au cœur des proverbes, comme stratégie argumentative, exemplifiée en particulier par l'entreprise de séduction.

\section{SYNTAXE DU SYSTEME HYPOTHETIQUE ET EFFETS DE SENS}

La diversité des emplois de si se résout, comme toujours en pareil cas, selon les approches linguistiques, en termes d'homonymie, de polysémie ou alors par la recherche d'un invariant de sens qui serait commun à l'ensemble des emplois. C'est cette dernière position qui est adoptée par exemple par les auteurs de la Grammaire méthodique du français: ceux-ci déduisent de la variété des emplois une fonction commune, celle de "poser ou plutôt présupposer le cadre situationnel, sans l'asserter comme fait particulier ${ }^{4}$; les valeurs diverses associées à $s i$ ne seraient que des « effets de sens », dépendants du contexte.

\section{I.1. Systèmes hypothétique et conditionnel ${ }^{5}$}

Syntaxiquement, si fonctionne comme marqueur d'une proposition au mode indicatif subordonnée à une proposition dont le mode est plus libre. La non-autonomie de la

\footnotetext{
$1 \quad$ Il ne faut jurer de rien (Jurer) ; Il faut qu'une porte soit ouverte ou fermée (Porte).

$2 \quad$ G. Moignet (1981) : 197.

3 Voir G. Corminboeuf (2007).

$4 \quad$ R. Riegel, J.-C. Pellat, R. Rioul (2009) : 853.

5 Sur le plan sémantique, d'autres structures syntaxiques prennent une valeur hypothétique sans adopter le schéma [si p, q] ; par exemple «Je vais lui écrire, ou que le ciel m'écrase !» (Porte, 92); ou encore les propositions avec la conjonction «quand»: «Quand même on ne ferait que s'imaginer qu'on aime, est-ce que ce n'est pas une chose charmante? » (Porte, 48); «Je l'aimerais, que la vengeance serait la plus forte » (Jurer, III, 1, 111). Cette étude se limite aux cas où si est présent.
} 
proposition introduite par si est un critère définitoire qui conduit à parler de système hypothétique. La relation est hiérarchique, syntaxiquement instaurée par le statut même de la subordonnée par rapport à la principale, mais sur le plan sémantique, des rapports divers s'établissent entre les deux propositions. Ceux-ci varient, selon les contextes, « de la cause à l'effet, du principe à la conséquence, de la condition au fait $»^{6}$.

À partir du corpus d'étude, un premier ensemble se dégage rassemblant les systèmes qui expriment soit une condition, soit une simple supposition ou éventualité. Le départ entre les différentes relations sémantiques n'est cependant pas toujours aisé à faire ; certains cas restent indécidables. Un exemple du type «Si vous êtes de bonne humeur, vous parlerez » $($ Porte, 28) peut être glosé par une phrase comme «vous parlerez parce que vous êtes de bonne humeur » ou «à la condition que vous soyez de bonne humeur» et être interprété comme un cas de proposition conditionnelle. La relation de cause à conséquence ou de «conditionnant à conditionné $»^{7}$ est claire également pour les systèmes qui prennent la forme d'une menace : «Je te déshérite, si tu refuses de te marier » (Jurer, I, 1,64); «Si vous n'y allez pas, je vous casse ma canne sur le dos » (Jurer, III, 1, 107).

On conservera cependant pour l'analyse le terme générique de proposition hypothétique dans un système corrélatif, en reprenant le classement de O. Ducrot. La relation est dite implicative quand le système est équivalent à [Si p, alors q] : si introduit une condition suffisante à la conséquence énoncée par $\mathrm{q}^{8}$. À cet emploi dit standard de $s i$, s'ajoutent des emplois où la valeur hypothétique de si est discutée. O. Ducrot évoque ainsi des emplois «marginaux» qu'il décline en quatre valeurs principales, le si oppositif, contrastif, austinien et présuppositionnel ${ }^{9}$, entrant dans des systèmes que d'autres appellent non conditionnels. Je retiendrai ici la vision unifiée des hypothétiques en posant que si, quel qu'il soit, établit un rapport discursif entre les deux contenus propositionnels énoncés et crée un univers de discours qui sert de cadre provisoire et de référentiel pour les énoncés ultérieurs ${ }^{10}$. L'analyse de Ducrot pour le si présuppositionnel, le cas le plus original de la théorie, peut être illustrée par l'exemple [1].

[1] Si le beau corps trouvé à Milo a jamais eu un modèle vivant, assurément cette grande gaillarde a eu plus d'amoureux qu'il ne lui en fallait, et elle s'est laissé aimer comme une autre, comme sa cousine Astarté, comme Aspasie et Manon Lescaut. (Porte, 49).

$S i$ introduit une proposition p qui constituerait le présupposé de q, si celle-ci était employée seule.

Ces différents systèmes se distinguent en outre par un critère distributionnel. Quand le système est non conditionnel, pour les exemples du corpus, la subordonnée ouvre le système. En revanche, quand la relation est d'ordre implicatif, l'ordre peut être inversé et la principale se trouver en position thématique. Les cas les plus nombreux du corpus placent la proposition subordonnée en position thématique, en ouverture du système ; la proposition [si p] répond ainsi à la fonction de cadre situationnel qu'on lui attribue. Dix exemples inversent cependant cet ordre attendu. La prosodie comme les modalités de phrase viennent interférer avec l'agencement des éléments. Si on n'examine que les phrases affirmatives où la proposition [si p] se trouve postposée, on peut se demander si la présence d'une pause syntaxique marquée

\footnotetext{
$6 \quad$ O. Ducrot (1991). Dire et ne pas dire : 167.

7 H. Vairel (1982).

$8 \quad$ O. Ducrot (1991). Dire et ne pas dire.

9 Sur l'exemple canonique du si austinien «si tu as soif, il y a de la bière dans le frigidaire », se calque l'occurrence du corpus «Et si vous vouliez rester à dîner, nous avons un faisan truffé ». (Jurer, II, 1, 87) qui s'insère dans la série d'invitations que Cécile lance à Valentin dans la scène 1 de l'Acte II. Ce type d'exemples est réfractaire à l'épreuve de la contraposition * si vous ne voulez pas rester, nous n'avons pas de faisan truffé.

$10 \quad$ O. Ducrot (1991). Dire et ne pas dire : 185.
} 
par une virgule est un critère discriminant ou pas. En présence d'une virgule, certains linguistes estiment que la place de la proposition subordonnée, avant ou après la principale, n'a pas d'incidence sur le sens de l'ensemble ${ }^{11}$. La proposition est circonstancielle et vérifie le critère de la mobilité de ce type de propositions, avant ou après la principale.

[2] Mais pensez donc que tout ce que je vous dis là, dans quatre ans d'ici un autre le fera, si j'épouse Mademoiselle de Mantes ; et comment voulez-vous que je sache de quelle résistance elle est capable, si je ne l'ai d'abord essayé moi-même ? (Jurer, II, 1, 80)

[3] Elle ne quitterait pas son piquet pour un prince ; mais que Dupré vienne, et qu'il lui parle bas, vous la verrez se lever de table, si c'est un mendiant qui attend. (Jurer, III, 4, 137)

[4] C'est qu'on fait un whist au salon, et que ma mère vous serait bien obligée si vous vouliez faire le quatrième. (Jurer, II, 1, 87)

Ce dernier exemple présente une absence de pause avant la proposition subordonnée. S. De Vogüe établit une typologie en trois volets : dans le dernier type, [si p] est obligatoirement postposé, sans pause et avec une marque d'accentuation sur $s i^{12}$. L'exemple [4] ne répond pas à cette description. En dépit de l'absence de pause, l'ordre des propositions ne paraît pas contraint et l'accentuation sur si est peu probable. La postposition encourage néanmoins la lecture intraprédicative de la subordonnée comme foyer de la phrase ${ }^{13}$, puisque cette tournure revient finalement à proposer à Valentin une partie de whist; ce n'est qu'un détour poli équivalent à l'interrogation directe «voulez-vous faire le quatrième ? ». Quoi qu'il en soit, l'agencement des propositions doit être évalué en contexte, comme choix raisonné et tactique. La postposition de la proposition subordonnée est l'ordre non canonique et doit se justifier. Dans l'exemple [2], la postposition redoublée joue en faveur de l'équilibre de la phrase organisée autour du «et » central et le jeu des tiroirs verbaux organise une argutie comme justification du stratagème de Valentin. La segmentation de la phrase place en clôture le mariage projeté, pivot de la pièce tout entière, tandis que l'interrogation directe ensuite fait passer Valentin, à quelques mots d'intervalle, du statut d'époux virtuel à celui d'amant, roué et impudent, effectif ; syntaxiquement, ce changement est soutenu par le passage du futur au passé composé, associé à l'aspect accompli.

Dans l'exemple [3], la symétrie de la phrase qui joue sur le contraste stéréotypé entre deux statuts (un prince, un mendiant) justifie la postposition de la subordonnée et sa place, de fait, à la fin de la phrase, à un poste-clé. Enfin, l'exemple [4] s'inscrit dans la continuité des interventions précédentes de Cécile qui dissimule ses demandes à Valentin sous forme de discours rapporté, attribué à sa mère ; à la forme de discours indirect « Monsieur, ma mère m'a chargée de vous demander si vous comptiez partir aujourd'hui », fait suite un fragment de discours indirect libre qui permet de placer au premier plan la «mère » comme un paravent derrière lequel s'abritent les désirs de Cécile.

Dans le même proverbe, la structure [q, si p] revient à trois reprises avec le même prédicat dans la principale, le verbe «épouser ». Près de la moitié des occurrences du verbe « épouser » que compte la pièce se concentre dans la scène 1 de l'Acte II, rythmée par les entrées et sorties de Cécile, et consacrée à l'exposé de l'épreuve imaginée par Valentin. Dans la protase, le verbe «épouser» est ainsi martelé comme l'idée fixe de l'oncle que Valentin s'ingénie à contrarier constamment.

11 S. de Vogüe (1992).

12 Du type «Je viendrai si j'ai le temps » : dans ce cas, la linguiste soutient que [si P] a le statut énonciatif de prédicat et énonce une condition nécessaire mais non forcément suffisante.

13 M. Charolles (2003). Voir un exemple similaire : «vous fâcherez-vous si je vous le dis ? (Porte, 29), détour pragmatique équivalent à une interrogation directe : «puis-je vous le dire ?». L'exemple avec présentatif dans la principale «voilà quatre louis si vous refusez » (Jurer, III, 1, 107) présente encore une subordonnée postposée, foyer de la phrase. 
[5] Tu l'épouseras si elle te reçoit mal ? (Jurer, II, 1, 81)

[6] Tu l'épouseras si elle persévère ? (Jurer, II, 1, 87)

[7] Épouse une blanchisseuse si tu veux. (Jurer, II, 1, 86)

L'équivoque relevée par O. Ducrot ${ }^{14}$ quand la principale est interrogative entre une structure implicative ou concessive (même si) est levée par le contexte. Dans les exemples [5] et [6], la relation entre les deux propositions est de type implicatif. Dans l'exemple [7], «si tu veux », fait figure d'expression quasi lexicalisée, répondant à la réanalyse d'une proposition subordonnée en une lexie, du type «s'il vous plaît » (Porte, 38$)$ ou même «si vous voulez » (Porte, 47$)$, « si vous m'en croyez » (Jurer, III, 1, 108).

\section{I.2. Systèmes non conditionnels et dialogisme}

Dans les systèmes non conditionnels, les deux contenus propositionnels sont validés en mémoire ; c'est leur potentiel dialogique ${ }^{15}$ qui les distingue le plus efficacement des systèmes conditionnels, "engageant une stratégie de contestation en modulant le domaine de validité d'un fait ${ }^{16}$. Les [Si p] extraprédicatives articulent, dans le contexte théâtral, une réplique à une autre. Leur fonction est cohésive ${ }^{17}$ et pragmatique.

[8] LA MARQUISE - Et vous, cela vous surprend donc bien de ce qu'on ose vous braver en face ?

LE COMTE - Prenez garde ! Si vous êtes brave, j'ai été hussard, moi, madame, je suis bien aise de vous le dire, et il n'y a pas encore si longtemps. (Porte, 40)

La réplique du Comte procède à un réajustement du verbe «braver » employé par la Marquise, qu'il remodèle en l'adjectif dont il dérive pour renchérir avec le nom « hussard ». L'adjectif «brave » cumule un emploi en usage et un emploi autonyme et joue, en contexte, en faveur d'un effet comique. Le glissement depuis le verbe «braver» jusqu'au nom «hussard» en passant par le relais de l'adjectif «brave», s'il est cohérent, joue sur les connotations du terme « hussard », proposant un renchérissement qu'on peut juger excessif.

Dans d'autres exemples, une relation explicative est marquée entre les deux propositions par des variantes en [Si p, c'est que/c'est pour/c'est parce que]. [Si p] est une forme nominalisée de $\mathrm{p}$, reprise par le démonstratif anaphorique dans q.

[9] Van Buck- Restez-vous ici, oui ou non?

Valentin - Si je reste, c'est pour notre gageure [...]

Valentin - [...] Il faut que j'écrive ; je vous reverrai à dîner.

Van Buck - Écrire ! J'espère que ce n'est pas à elle que tu écriras.

Valentin - Si je lui écris, c'est pour notre gageure. Vous savez que c'est convenu. (Jurer, II, 1, 89-90)

Sur le plan syntaxique, ces structures sont à rapprocher des phrases clivées du type «c'est pour notre gageure que je reste » sans en être les équivalents sur le plan pragmatique ; cet échange est particulier en ce qu'il ne répond pas à la structure canonique des exemples de ce type. En effet, le premier membre n'a pas été l'objet d'une assertion antérieure, d'un fait qui aurait déjà été validé en mémoire. Ces systèmes sont des réponses détournées à l'interrogation précédente. À la demande pressante exprimée par l'interrogation directe totale, le neveu

\footnotetext{
$14 \quad$ O. Ducrot (1991): $171 s q$.

15 M. Monte (2009) : 99.

16 G. Corminboeuf (2009) : 343.

17 La proposition extraprédicative, détachée à gauche assure une fonction de cohésion discursive : elle (re)topicalise un contenu de cotexte gauche et fait contraste avec q. Voir Achard-Bayle (2009) : 4.
} 
répond par un système hypothétique qui dévie la réponse assertive sur la cause. Le foyer de la proposition se déplace sur la cause et élude la réponse directe, en position thématique.

Le déroulement de l'échange suivant adopte une stratégie similaire, tout en déclinant un polyptote organisé autour du verbe «écrire ». La réplique de Van Buck qui s'ouvre sur le modalisateur «j'espère » fonctionne comme variante optative de la demande d'information, tout en orientant l'interrogation dissimulée vers le pôle négatif de la réponse. La réponse du neveu est déceptive là encore car elle est contournée par le relais du système hypothétique causatif; l'apodose entre en écho avec la précédente réplique; le possessif «notre gageure » est, plus qu'un appel à la connivence, une façon d'enrôler l'allocutaire dans une compromission imparable.

Sur le plan sémantique, les systèmes hypothétiques instaurent un mouvement suspensif soutenant un échange qui se construit en co-énonciation.

\section{SYSTEMES HYPOTHETIQUES ET CO-ENONCIATION}

\subsection{Un mouvement suspensif}

Les linguistes ont montré la proximité de l'interrogation et de l'hypothétique ${ }^{18}$, qui ont en commun de ne pas être assertées et de «suspendre la valeur de vérité de la proposition [qu'ils introduisent] $»^{19}$. On sait que si se partage entre un emploi intégratif et un emploi percontatif $^{20}$. «La phrase interrogative est certes indéterminée au regard de la vérité. Elle apparaît comme un au-delà par rapport au vrai et au faux, comme une fonction suspensive de la valeur de vérité, comme la mise en débat d'une proposition préalablement envisagée dans quelque image d'univers comme vraie et comme fausse ${ }^{21}$. Les formes non conditionnelles des hypothétiques sont, de même, analysables par une mise en doute fictive de la réalité.

Quand il introduit une interrogative indirecte ou percontative, si pose la question de la vérité de la proposition; la portée de l'interrogation est totale en ce sens qu'elle attend une réponse par oui ou non ${ }^{22}$.

[10] Je veux jeter cette pièce à pile ou face pour savoir si je l'aimerai. (Jurer, II, 1, 91)

L'interrogative indirecte tourne ici en dérision le sentiment amoureux comme Valentin le fait encore avec des systèmes hypothétiques qui construisent une vision simplifiée et binaire de la réalité, reposant sur deux alternatives contraires uniques et explicitées :

[11] Si elle tourne la tête de notre côté, je l'aime ; sinon je m’en vais à Paris. (Jurer, II, 1, 91)

[12] Je lui dirai que [...] si elle me repousse, je me brûle la cervelle, et que si elle veut de moi, je l'enlève demain matin. (Jurer, II, 1, 92)

La concentration de ces exemples dans la même scène, à quelques lignes d'intervalle, joue en faveur du comique qui table sur la légèreté frivole qu'affiche Valentin. Le sentiment amoureux est réduit à une mécanique qui se pare, de manière parodique, des excès du romanesque dans la citation [12].

\subsection{Les univers alternatifs}

Les deux contenus propositionnels se trouvent placés dans le même monde créé par si. Cet univers est un monde parallèle au monde factuel. Si peut donc ouvrir à des mondes contrefactuels, que les grammaires nomment l'irréel du présent ou l'irréel du passé.

\footnotetext{
$18 \quad$ Voir par exemple M. Wilmet (1997) : $\$ 634,3^{\mathrm{e}}$.

$19 \quad$ R. Martin (1983) : 98.

$20 \quad$ P. Le Goffic $(1993): \S 288$.

$21 \quad$ R. Martin $(1987): 21$.

22 L'exemple «je vous demande si on va boire ça. » (Jurer, III, 2, 114) se situe entre interrogative et assertive. En fait, l'interrogation oriente vers la réponse négative.
} 
L'hypothèse est fausse en regard de la vérité objective mais fait entrer dans la vérité d'un monde imaginaire, qui sert de relais pour éclairer le monde « réel ». De fait, la construction de mondes alternatifs n'est qu'un détour pour parler du monde où les personnages se trouvent ; ce sont des boucles qui ramènent toujours au monde contingent mais en y enfermant l'allocutaire. Si ouvre un «référentiel possible ${ }^{23}$ en rupture avec le référentiel énonciatif.

[13] La Marquise - Il me semble, en vérité, que, si j'étais homme et si je voyais une jolie femme, je me dirais : Voilà une pauvre créature qui doit être bien assommée de compliments. Je l'épargnerais, j'aurais pitié d'elle, et, si je voulais essayer de lui plaire, je lui ferais l'honneur de lui parler d'autre chose que de son malheureux visage. (Porte, 35$)$

[14] La Marquise - Si j'avais une fille, et si je voulais la préserver de ces entreprises qu'on appelle dangereuses, je me garderais bien de lui défendre d'écouter les pastorales de ses valseurs. (Porte, 45)

Les exemples des propos de la Marquise présentent des hypothèses enchâssées. «Si j'étais homme » et «si j'avais une fille» énoncent des propos en contradiction flagrante avec la réalité objective ou le monde que le locuteur admet comme le monde de ce qui est $^{24}$. À cette première hypothèse contrefactuelle se greffe une deuxième supposition, plausible dans l'univers ainsi créé.

L'exemple [15] propose un exemple d'irréel du passé :

[15] La Marquise - Si vous aviez trouvé ici trois personnes, les premières venues, là, au coin de ce feu, vous parleriez, à l'heure qu'il est, littérature ou chemins de fer, après quoi vous iriez dîner. (Porte, 42)

À l'intérieur des univers de croyance se déploient deux mondes possibles, potentiels ou contrefactuels quand une au moins des propositions est en contradiction avec le monde effectif. L'irréel du passé décrit une situation qui aurait pu se réaliser dans le passé et signale la contradiction entre ce qui s'est réalisé dans le passé et un des possibles dans le passé, avec les incidences éventuelles dans le présent d'énonciation ; l'irréel du présent dénote une situation fausse eu égard au monde effectif, qui sert de parcours imaginaire en vue d'une démonstration. Cela suppose une vision ramifiée du temps.

Ces propositions en [si p] sont directement orientées vers l'allocutaire que l'énonciateur entraîne dans ses suppositions et ses constructions alternatives.

L'énonciation d'une conditionnelle implique que le rédacteur et le lecteur s'accordent (provisoirement) pour sélectionner un état de choses (spécifié par la protase) sur un ensemble d'états de choses supposé accessible dans le contexte, et cela à la seule fin d'accéder à un nouvel état de choses qui est exprimé par $1^{\prime}$ apodose ${ }^{25}$.

Le but est, pour la Marquise en particulier, de construire un univers parallèle qui puisse servir de modèle au comportement du Comte, qu'elle feint de fustiger par le contraste qu'elle installe entre les deux univers. Les mondes parallèles imaginés sont co-construits, visant la coïncidence entre le monde factuel et le monde imaginaire, développée dans l'apodose des systèmes.

Les hypothèses construites acquièrent la réalité des paroles prononcées. Leur existence est intangible dans le monde verbal qui tisse des liens indissolubles avec le monde « réel ». La mémoire discursive, conçue comme «ensemble évolutif de représentations partagées qui s'élabore publiquement et coopérativement au long du discours ${ }^{26}$ se nourrit des mondes possibles ouverts par les si qui reconfigurent le réel.

L'enjeu pragmatique des systèmes hypothétiques récurrents joue comme ressort dramatique dans les Proverbes envisagés.

$\begin{array}{ll}23 & \text { J.-P. Desclés (1994). } \\ 24 & \text { R. Martin }(1987): 16 . \\ 25 & \text { M. Charolles }(2003): 26 . \\ 26 & \text { G. Corminboeuf, Grammaire de la période, II, à paraître. }\end{array}$




\section{ARGUMENTATION ET SEDUCTION}

Vous allez à droite, vous regardez à gauche; vous allez à gauche, vous regardez à droite; il n'y a rien de plus naturel. (Jurer, I, 2, 70)

Les systèmes hypothétiques fonctionnent comme des détours énonciatifs, dont le modèle figuré pourrait être cette description de la valse, par le maitre de danse, donnée en exergue à cette partie. La communication est indirecte; les boucles hypothétiques sont le meilleur moyen que trouvent les personnages pour atteindre leur but.

\subsection{Les systèmes hypothétiques incomplets : Dérobade et esquive}

Les systèmes hypothétiques sont quelquefois incomplets mais il faut faire la part entre les systèmes interrompus ou suspensifs et ceux qui, même s'ils ne présentent que la proposition en $s i$, n'en sont pas moins autonomes et complets.

Ces derniers répondent à un schéma intonatif spécifique et se coulent dans le moule de la phrase exclamative à la mélodie descendante. «Encore si » fonctionne comme marqueur d'une clause ou unité syntaxiquement autonome.

[16] Van Buck - Encore si tu étais amoureux ! si je pouvais croire que tant d'extravagances partent d'un motif qui eût quelque chose d'humain ! Mais non, tu n'es qu'un Lovelace, tu ne respires que trahisons, et la plus exécrable vengeance est ta seule soif et ton seul amour.

Valentin - Encore si je vous voyais pester ! si je pouvais me dire qu'au fond de l'âme vous envoyez cette baronne et son monde à tous les diables ! Mais non, vous ne craignez que la pluie. (Jurer, III, 1, 109)

Ces phrases sont indépendantes. Elles inscrivent l'énoncé contraire en filigrane en le doublant d'une modalisation dépendante du contexte : il peut s'agir d'un regret, d'un conseil, d'une requête par exemple. Dans l'exemple [16], la réplique de Valentin singe celle qui précède de son oncle ; toutes les deux orientent vers les assertions négatives correspondantes : «tu n'es pas amoureux ; je ne peux croire » et «je ne vous vois pas pester; je ne peux pas dire », se situant cependant entre regret et sollicitation pour un changement d'attitude adressée à l'allocutaire.

En revanche, le schéma est suspensif quand le système prend la forme d'une interrogation ou lorsque la proposition en si est suivie d'un point de suspension justement :

[17] La Marquise - Avertissez au moins : est-ce une déclaration, ou un compliment de bonne année ?

Le Comte - Et si c'était une déclaration? (Porte, 33)

L'hypothèse est amorcée par la proposition en si et laisse l'allocutaire libre de compléter le conséquent de la protase, premier élément d'un système incomplet, auquel la Marquise répond par une pirouette. «Oh ! c'est que je n'en veux pas ce matin ». De la part du Comte, c'est un aveu du bout des lèvres qui se réserve l'échappatoire de l'énoncé négatif correspondant puisque celui-ci n'est pas écarté comme il le serait avec un énoncé assertif «c'est une déclaration ». L'imparfait véhicule des connotations d'éventualité, dérive modale attachée à l'aspect sécant de ce tiroir verbal ; de fait, l'univers ouvert par l'acte de supposition réalisé par si est toujours révocable car accepté comme cadre provisoire du discours ${ }^{27}$; les deux options de l'interrogation, négative et affirmative, sont préservées.

Un peu plus loin dans la pièce, la formule suspensive «Si vous le disiez sérieusement...» (Porte, 39) exprime le regret du Comte face à la froideur affichée de la Marquise, tout en exprimant une requête dissimulée, équivalent pragmatique de «dites-le sérieusement ».

L'interruption d'un système hypothétique est l'occasion de jeux scéniques dans le duo-duel formé par la Marquise et le Comte :

27

O. Ducrot (1991) : 167. 
[18] Le Comte.- Les paroles d'un homme qui vous aime vous font l'effet d'un méchant roman ? Ses regards, ses gestes, ses sentiments vous semblent une comédie ? [...]

Le Comte. - [...] Eh ! mon Dieu ! si l'amour est une comédie...

La Marquise. - Le feu ne va pas : la bûche est de travers

Le Comte, arrangeant le feu. - Si l'amour est une comédie, cette comédie, vieille comme le monde, sifflée ou non, est, au bout du compte, ce qu'on a encore trouvé de moins mauvais. (Porte, 46-47).

La réplique de la Marquise qui interrompt le Comte peut faire figure d'apodose du système initié par le Comte. Dès lors, la rencontre apparemment fortuite de ces deux segments reconstitue un système hypothétique tout entier en assurant la complétude syntaxique dont on peut déduire la complémentarité sémantique. Une double lecture de la réplique de la Marquise est alors possible : on peut y lire une réplique banale, acte de langage indirect qui invite le Comte à s'occuper du feu, ou alors une expression métaphorique dont l'interprétation reste ouverte, mais qui referme sémantiquement le système hypothétique, avant la reprise en main par le locuteur premier.

Dans ce Proverbe, La Marquise et le Comte se livrent à une joute verbale animée par les jeux de la séduction.

\subsection{Jeu de séduction : les compliments détournés}

Les deux proverbes reposent sur une entreprise de séduction; le terme est pris en mauvaise part dans Il ne faut jurer de rien ${ }^{28}$; il prend davantage les atours du badinage dans Il faut qu'une porte soit ouverte ou fermée.

Les systèmes hypothétiques participent de ce jeu qui est aussi un jeu rhétorique. C'est par exemple l'occasion d'une réorientation discursive par l'interlocuteur qui reprend en main le cadre posé par l'autre.

[19] Le Comte - Je ne sais pas, mais je vous ennuie, vous me le disiez vous-même tout à l'heure [...]

La Marquise - Si je vous ai dit que vous m'ennuyiez ce matin, c'est que ce n'est pas une habitude. (Porte, $37)$.

La Marquise joue sur l'identification du prédicat, qui ne coïncide pas avec le prédicat verbal dans sa réponse, mais avec le circonstant temporel « ce matin »; la portée du verbe est réduite à un espace temporel ponctuel. C'est une manière de compliment qui affirme que la Marquise trouve plaisir à recevoir le Comte.

Le compliment se dissimule sous une apparence d'argumentation lorsque le Comte emploie un système non conditionnel, qui maintient le présent de l'indicatif dans la protase et l'apodose :

[20] Si vous ressemblez à votre grand-mère, est-ce que vous en êtes moins jolie ? (Porte, 49).

Le système hypothétique se présente comme un exemple de plus dans la réponse du Comte à la Marquise qui se plaint des ritournelles monotones des discours galants. La répétition dans les formules ne prive pas le sentiment amoureux de sa beauté et de son éternelle jeunesse, avance le Comte. L'habileté du Comte est d'inscrire cette allusion au charme de son interlocutrice dans une série, qui fait dévier de la beauté du sentiment à la beauté physique de la Marquise en passant par le relais symbolique de Vénus, la déesse de l'amour. Le tour interrogatif dans l'apodose du système hypothétique s'assimile à une interrogation oratoire qui équivaut, par inversion de polarité, à une assertion renforcée : "vous êtes très jolie ». L'ensemble s'inscrit dans une stratégie argumentative qui n'a d'autre but que de dissimuler une déclaration. La Comtesse cependant ne s'y trompe pas mais c'est pour balayer le

$28 \quad$ N'est-ce donc vraiment qu'une séduction que vous venez tenter ici sous le masque de cette épreuve ? (Jurer, II, 1, 84). Le terme a ici le sens étymologique. 
compliment comme flagornerie de séducteur, en reprenant l'adjectif en mention : « Bon, voilà le refrain : jolie » (Porte, 49$)$.

C'est encore avec des si que le Comte avance à demi-masqué pour complimenter la Marquise et se déclarer son dévoué :

[21] Le Comte - Ce ne sera pas par hasard, madame, si je puis vous être bon à quelque chose.

La Marquise - Encore un compliment ! Mon Dieu, que vous m'ennuyez ! (Porte, 35-36)

La tournure s'apparente à une structure disloquée. La structure phrastique amorcée par si est annoncée par le démonstratif «ce » et commutable avec une proposition en c'est...que (ce ne sera pas par hasard que je pourrai vous être bon à quelque chose) qui l'oriente vers une percontative $^{29}$; la réalité dénotée dans la proposition en si est mise fictivement en doute en vue d'un dévouement affirmé.

[22] S'il était possible, ce serait ma vie entière que je mettrais à vos pieds ; ce serait mon nom, mes biens, mon honneur même que je voudrais vous confier. (Porte, 52).

Avec l'exemple [22], les tiroirs verbaux de l'imparfait et du conditionnel présent orientent vers l'interprétation de l'énoncé comme irréel du présent. On constate cependant une disproportion entre le membre subordonné en si et l'apodose à la structure emphatique redoublée, qui s'étire en énumérant les sacrifices que le Comte est prêt à faire à celle qu'il courtise, «ma vie entière, mon nom, mes biens, mon honneur » avec un effet de gradation rendu par l'adverbe «même » pour le dernier élément. La brièveté de la conditionnelle à l'initiale de la phrase tend à s'estomper face au volume de l'apodose. L'ensemble peut ainsi sonner comme véritable déclaration d'un amour absolu.

\section{CONCLUSION}

Les $s i$ qui interviennent dans les systèmes hypothétiques, qu'ils soient conditionnels ou non, se rejoignent par leur capacité à amorcer un mouvement suspensif, à la fois sur le plan syntaxique pour les systèmes corrélatifs complets et sur le plan sémantique et pragmatique. Ces systèmes ont une dynamique qui les oriente vers l'allocutaire, entraîné dans la coconstruction de mondes alternatifs, posés comme cadres discursifs préalables.

L'enjeu pragmatique des systèmes [Si p, q] est utilisé comme ressort dramatique dans les deux pièces de Musset. Manifestant un détournement, un écart par rapport à la ligne droite du discours, ces systèmes servent une stratégie d'évitement et d'esquive. Valentin qui joue au roué s'en sert pour enfermer son oncle dans des boucles ratiocinantes afin d'échapper à la perspective du mariage imposé. Le Comte et la Marquise construisent ensemble des mondes alternatifs et hypothétiques qui permettent d'atteindre la réalité de manière indirecte et biaisée, tout en respectant les conventions sociales.

Au-delà des intrigues particulières, les proverbes décrivent les relations hommes-femmes comme un parcours sinueux où le doute est omniprésent. C'est sans doute la fragilité humaine, la peur face au sentiment amoureux qui peuvent se lire au travers des hypothétiques qui se multiplient et qui servent de paravents à la déception éventuelle. Le titre même Il faut qu'une porte soit ouverte ou fermée est emblématique des tergiversations des personnages, dont les propos se situent toujours dans un entre-deux. Quand la déclaration du Comte aura été acceptée par la Marquise, la porte pourra être refermée définitivement et c'est la fin de la pièce. Dans Il ne faut jurer de rien, le proverbe est lancé dès le titre, ouvrant un mouvement suspensif, comme une hypothèse à valider, le temps de l'intrigue. La réplique finale de Valentin, qui reprend en écho ce proverbe en l'appliquant à un cas particulier, assure le

$29 \quad$ P. Le Goffic (1993) : 409. On peut conserver cette analyse pour les énoncés du type «À peine si le sable garde l'empreinte de nos pas; le gravier sec a déjà bu la pluie » (Jurer, III, 3, 118), en supposant l'ellipse de « c'est à peine si.... ». 
bouclage du système. Le transfert d'une énonciation générique à un contexte énonciatif particulier oriente les inférences à déduire du proverbe. Les relations sociales et amoureuses se dessinent comme un lacis de lignes qui se déploient, le temps des proverbes, pour finir par se refermer par le choix d'une alternative, par la résolution des possibles quand une seule hypothèse est validée.

\section{BIBLIOGRAPHIE}

Corpus

Musset

Il ne faut jurer de rien [1836], Paris, Gallimard, 2011.

Il faut qu'une porte soit ouverte ou fermée [1845], Paris, Flammarion (2003).

\section{Bibliographie critique}

ACHARD-BAYLE G. (2009), Détachement topical et organisation en écho du texte : le cas des « Si $\mathrm{p} »$ contrastives in Apothéloz D., Combettes B. et Neveu F. (éds). Les Linguistiques du détachement, actes du colloque de Nancy, juin 2006, Berne, Peter Lang, p. 3-19.

ADAM J.-M. (1991). Langue et littérature, Paris, Hachette.

CHAROLLES M. (2003), "De la topicalité des adverbiaux détachés en tête de phrase », Travaux de linguistique, $\mathrm{n}^{\circ}$ 47, p. 11-51.

CORMINBOEUF G. (2010), «Une description des constructions introduites par un si dit austinien. Travaux de linguistique, $\mathrm{n}^{\circ}$ 60, p. 127-144.

CORMINBOEUF G. (2009), L'expression de l'hypothèse en français. Entre hypotaxe et parataxe. Bruxelles, De Boeck.

CORMINBOEUF G. (2007), «Coordination subordination, corrélation ou énonciation autonome », TRANEL, ${ }^{\circ}$ 47, p. 117-194.

DESCLÉS J.-P. (1994), «Quelques concepts relatifs au temps et à l'aspect pour l'analyse des textes », Studia Kognitywne, Semantyka kategorii Aspektu i czasu, n ${ }^{\circ}$ 1, Polska Akademia Nauk, Institut Slawistiki, p. 57-88. http://lalic.paris-sorbonne.fr/PUBLICATIONS/descles/sevres1.pdf

LE GOFFIC P. (1993), Grammaire de la phrase française, Paris, Hachette.

DE VOGÜE S. (1992), «Si, la syntaxe et le point de vue des opérations ». In Théorie d'Antoine Culioli, Paris, Ophrys, 1992, p. 123-144.

MARTIN R. (1987), Langage et croyance. Les univers de croyance dans la théorie sémantique, Bruxelles, Mardaga.

MARTIN R. (1983), Pour une logique du sens, Paris, PUF.

MOIGNET G. (1981), Systématique de la langue française, Paris, Klincksieck.

MONTE M. (2009), «Si marqueur d'altérité énonciative dans les si p extraprédicatives non conditionnelles », Langue française, $\mathrm{n}^{\circ}$ 163, Dialogisme et marqueurs grammaticaux, p. 99-119.

RIEGEL M., PELLAT J.-Ch., RIOUL R. (2009). Grammaire méthodique du français, PUF.

VAIREL H. (1982), «Les phrases conditionnelles/hypothétiques en français : la valeur de si $\mathrm{A}, \mathrm{B} », L^{\prime}$ Information grammaticale, $\mathrm{n}^{\circ} 14$, p. 5-10. http://www.persee.fr/web/revues/home/prescript/article/igram_0222-9838_1982_num_14_1_2349

WILMET M. (1993), Grammaire critique du français, Louvain-la-Neuve, Duculot. 


\section{Résumé}

L'enjeu de l'article est d'articuler l'analyse linguistique, syntaxique et sémantique, des systèmes hypothétiques [Si p, q] dans deux Proverbes de Musset, Il faut qu'une porte soit ouverte ou fermée et Il ne faut jurer de rien, et l'interprétation stylistique, afin d'en découvrir les enjeux argumentatifs et dramatiques. L'instauration d'un mouvement suspensif construit un échange en co-énonciation; la construction de mondes alternatifs et parallèles au monde factuel permet aux personnages d'atteindre la réalité par le détour hypothétique. 